\title{
A Comprehensive Analysis for the Heterogeneous Effects on Driver Injury Severity in Single-Vehicle Passenger Car and SUV Rollover Crashes
}

\author{
Huiying Wen $\mathbb{D}^{1},{ }^{1}$ Zuogan Tang $\mathbb{D}{ }^{2}$ Yuchen Zeng, ${ }^{1}$ and Kexiong Zhang ${ }^{1}$ \\ ${ }^{1}$ School of Civil Engineering and Transportation, South China University of Technology, Guangzhou, China \\ ${ }^{2}$ Department of Intelligent Transport, Shenzhen Urban Transport Planning Center, Shenzhen, China \\ Correspondence should be addressed to Zuogan Tang; zuogantang@163.com
}

Received 26 July 2019; Revised 24 October 2019; Accepted 4 November 2019; Published 13 January 2020

Academic Editor: Richard S. Tay

Copyright (c) 2020 Huiying Wen et al. This is an open access article distributed under the Creative Commons Attribution License, which permits unrestricted use, distribution, and reproduction in any medium, provided the original work is properly cited.

\begin{abstract}
In road traffic crashes, although rollover crashes account for a relatively low proportion, those result in a high fatality rate. The present study performed random parameters ordered logit models to examine risk factors as well as their heterogeneous effects on driver injury severity in single-vehicle passenger car and SUV rollover crashes. Crash data for the empirical analysis were extracted from Texas Crash Record Information System (CRIS) database during the year 2016. Model estimation results show that six variables (male drivers, drivers' age, airbag deployment, failure to drive in single lane, speed limit, and rural area) were found to produce normally distributed parameters in passenger car model, while nine parameters (male drivers, safety belt use, airbag deployment, drug or alcohol use, failure to drive in single lane, improper evasive action, vehicle model year, friday, and rural area) in SUV model were found to be normally distributed. Several other factors with fixed parameters were found to be associated with driver injury severity in single-vehicle passenger car or SUV rollover crashes, most notably: ejection or partial ejection, turning left, intersection, August, adverse weather conditions, and night with light. These variables were significant in both models; most variables have stronger effects on nonincapacitating injury and serious injury outcomes in SUV than in passenger car rollover crashes. These findings provide a deep insight into causality nature and factor involved in driver injury severity in single-vehicle passenger car and SUV rollover crashes and are also helpful for transport agencies to determine appropriate countermeasures aimed at mitigating injuries sustained by drivers in single-vehicle rollover crashes.
\end{abstract}

\section{Introduction}

Road traffic accidents are the major cause of fatalities and injuries globally. In a worldwide scale, the World Health Organization released that nearly 1.25 million citizens died of road traffic crashes and nearly 50 million individuals suffering nonfatal injuries as a consequence of such accidents [1]. Among these accidents, a large number of automotive vehicle accidents lead to vehicle rollovers (e.g., in the United States, $18.9 \%$ fatal crashes involved rollovers during the year 2014) and rollovers are becoming one of the crucial safety threats in road traffic events [2]. A rollover event is defined as [3]: "a vehicle involved in a rotation with at least 90 degrees in longitudinal axis level, regardless of whether the vehicle tips over onto its side or roof." Although rollover crashes constitute a small share of total traffic accidents, the number of rollover accidents and fatalities are out of proportion. In China, there were 5,036 rollover crashes occurring in 2016, which constituted only $2.68 \%$ of the total motor vehicle crashes, but caused $5.64 \%$ of all fatal accidents [4]. In the State of Texas, US, rollover crashes made up $6.70 \%$ of all traffic accidents during the year 2016, while the death toll caused by rollovers were responsible for $14.98 \%$ of total fatalities [5]. These statistics indicate that the number of casualties caused by rollovers is far from negligible, and the urgent need of taking in-depth insight into rollover crashes to mitigate their injury severity.

In the recent years, many efforts have been taken to analyze rollover crashes. Among these studies, quantities of studies mainly focused on the occurrence propensity or the frequency of rollover crashes, especially on those which involve specific vehicle types such as truck $[6,7]$, sport utility vehicle [8], passenger car $[9,10]$, tractor $[11,12]$, and tactical 
vehicle [13]. For instance, Farmer and Lund [6] observed that curved roadways in rural areas had a higher risk of resulting in rollovers and that drivers aged less than 25 years and light trucks were related to a higher probability of getting involved in rollover crashes. Khattak et al. [7] performed a binary probit model to explore truck rollover propensity. The results revealed that risky behavior driving, improper turning action, and curves were more likely to result in rollovers. From this study, [11] found that more than $2 / 3$ of the fatal tractor-related rollover crashes involved nonROPS (rollover protective structure) equipped vehicles, while only $16.6 \%$ of fatal tractor-related rollover crashes involved a clear environmental factor. Using seven count-data models, [13] observed that the light-vehicle traffic (LVT) was positively associated with the crash frequency, while the heavy-vehicle traffic (HVT) were found to have the opposite effect. In an interesting study, Reference [14] studied occupant injury patterns in high-mobility multipurpose wheeled vehicle (a type of tactical vehicle) rollover from 1992-2013, and reported that $56 \%$ of the occupant fatalities could have been prevented by restraint use. The summarized results from these studies indicate that aggregating different vehicle types (e.g., passenger cars, pickup trucks, SUV's) into one single model when analyzing rollover crashes may be inappropriate due to their differences in vehicle stability and crashworthiness.

Alternatively, some studies have addressed the issue of injury severity in rollover crashes. Conroy et al. [15] employed logistic regression models to predict serious injury of rollover crashes, using 27 severely injured occupants and 606 control occupants without injury or slight injury outcomes. Based on the multinomial logit model estimation results, $\mathrm{Hu}$ and Donnell [16] reported that the gradient of cross-slope and the width of median affected injury severity levels of median rollover accidents. Chen et al. [17] applied support vector machine models to explore contributing factors affecting driver injury severity and confirmed that comfortable driving conditions, alcohol/drug use, safety belt use, roadway lanes, driver attributes, vehicle damage severity, crash characteristics were associated with severe injury outcomes and fatality in rollover crashes. Wu et al. [18] found that wet pavement surface conditions, and alcohol involvement increase injury severities of rollover accidents. Reference [19] analyzed SUV and pickup truck rollover crashes using mixed logit models and verified that separating vehicle types for analysis rollover crash injury severity is necessary. More recently, using the random effects generalized ordered probit model, Anarkooli et al. [20] found that poor light conditions, adverse weather conditions, light truck and heavy vehicles, vehicle actions, vehicle mode year, traffic volumes, number of travel lanes, speed limits, and unsafe roadside conditions were related to severe injury levels in rollover crashes. Nevertheless, most of those studies failed to consider the unobserved individual heterogeneity which may affect injury severity of rollover crashes across observations.

Methodologically, there has been great interest in approaches that can capture unobserved heterogeneity in modeling crash injury severity. Various unobserved heterogeneity modeling approaches, such as random parameters probability models [21-23], latent-class logit models [24, 25], and Markov-switching models $[26,27]$ were employed to analyze crash injury severity over the years. Among these models, the random parameters approach which captures individual heterogeneity through relaxing parameters to vary across observations [21], is the most prevalent method. To the authors' knowledge, there have been few studies which applied the random parameters logit model to examine the rollover crash injury severity and analyzed the heterogeneous impacts of risk factors on driver injury outcomes in rollovers. As verified by previous studies, unobserved heterogeneity among some other risk factors may affect injury-severity outcomes, such as age [21], pavement conditions [28], roadway alignment [29], etc. In addition, the random parameters logit model cannot consider the order nature of injury severity crash data and thus such information might not be used to the full. Therefore, this paper aims to extend the previous study [18] and assumes that unobserved heterogeneity potentially exists in all explanatory covariates used in the next section on severity outcomes of single-rollover crashes and utilizes the random parameters ordered logit model to determine injury severity of rollover crashes.

As noted above, in the case of analyzing rollover crashes, separating vehicle types for analysis is more appropriate and comparable. Khattak and Rocha [8] found that passenger cars were less likely to be involved in rollovers compared with sport utility vehicles, probably due to that motor vehicles with light weight and small size are less prone to roll over. Similarly, Reference [19] reported that SUVs are almost twice as likely to be involved in fatal rollover crashes as passenger cars. Regarding the purpose and function, both SUVs and passenger cars are mainly used to transport people rather than goods and are private automobiles in most instances. Given the above, a conclusion can be made that the SUVs and passenger cars share common features on the purpose, but they have much difference in the injury patterns of rollover crashes. Therefore, it is important to explore the similarities and differences of the injury mechanism between SUV and passenger car rollover crashes for improving SUV and passenger car safety. In addition, the causality nature and factors involved are substantially different between singleand multi-vehicle rollover crashes. It appeals that separating these two crash types for empirical analysis is more rational, which is better to isolate specific vehicle and driver effects. Moreover, according to the data sample, single-vehicle rollover crashes responsible for more than $90 \%$ of all rollover crashes. Thus, the present study specifically focuses on analyzing single-vehicle passenger car and SUV rollover crashes separately.

To this end, the main objective of the study is to develop random parameters ordered logit models for analyzing driver injury severity in single-passenger car and SUV rollover crashes that accounts for heterogeneous impacts of risk factors across observations. Accordingly, the rest of this study is structured as follows. Section 2 shows crash dataset and a brief description. Section 3 introduces the proposed approach for modeling crash injury severity. Section 4 shows detailed parameters estimation results and discusses it. Some safety 
implications are present in Section 5. Lastly, conclusions and recommendations for future research are shown in Section 6.

\section{Data}

The single-vehicle rollover crashes during the year 2016 were extracted from Texas Crash Record Information System (CRIS), published by the Texas Department of Transportation. The CRIS database contains three major sub-database including accident, vehicle, and primary person. The accident sub-database describes each accident, including speed limit, weather conditions, roadway type, roadway alignment, surface conditions, traffic control, the first harmful event, manner of collisions, crash date, crash time, crash location, total injuries, day of week, level of severity. The vehicle subdatabase contains each vehicle involved in the accident, including vehicle type, factors for the vehicle contributed to the crash, vehicle type, vehicle action, model year. The primary person file reports the information of casualty involved in the crash, including person type, severity of injury, age, gender, ethnicity, ejection, restraint use, airbag deployment, alcohol involvement, drug involvement, license information.

In the data sample, the variable "THE_Collsn_ID", is used to distinguish collision types. Where the value of " 10 " means that the crash type is rollover. Likewise, the variable "Unit_ Nbr" represents the quantity of motor vehicles involved in collision. It is single-vehicle rollover crash when "THE_ Collsn_ID" equals "10" and "Unit_Nbr" equals "1". Two types of four-wheel motor vehicles including passenger car, and sport utility vehicle (SUV) were considered in the present analysis. A one-year dataset was used because it ensures a sufficient sample size, and meanwhile the short time span is more likely to control changes for other factors (e.g., road network structure, traffic volumes).

After data processing (eliminating unreasonable and missing data), there are 2400 single-passenger car and 2360 single-SUV rollover observations in total for injury severity modeling in the present study. According to the CRIS protocol, five-level scheme for crash injury severity is categorized as: "no injury", "possible injury", "nonincapacitating injury", "incapacitating injury" and "fatality". Driver injury severity is the outcome of injury sustained by the most severely injured driver in a vehicle. In order not to cause ambiguity, it is needed to provide definitions of possible injury and nonincapacitating injury. Possible injury is defined as injury claimed, reported, or indicated by behavior but without visible wounds, includes limping or complaint of pain. While nonincapacitating injury is evident injury such as bruises, abrasions, or minor lacerations which do not incapacitate [30]. Due to the low proportion of fatality in rollover observations, the incapacitating injury and fatality categories were combined into a single serious injury category. Therefore, in the present paper, analysis of injury severity is based on a four-level scheme: no injury, possible injury, nonincapacitating injury, and serious injury. The definitions and summary statistics of the variables used for driver injury severity modeling are shown in Table 1 . It is noteworthy that many previous studies modeled driver age, vehicle model year, and speed limit as continuous variables [31-33], while this paper treated them as categorical ones in order to minimize information loss.

\section{Methodology}

3.1. Crash Injury Severity Modeling. In the present study, the random parameters ordered logit mode is developed for driver injury severity modeling. Following previous studies conducted by Chang et al. [31], a linear function for injury propensity is defined as,

$$
S_{i}=\boldsymbol{\beta} \mathbf{X}_{i}+\varepsilon_{i},
$$

where, $S_{i}$ is a latent variable that determined the injury severity propensity for driver $i . \mathbf{X}_{i}$ is the explanatory variable set (e.g., drivers' age, safety belt use, airbag deployment, ejection or partial ejection, alcohol or drug involvement) associated with $S_{i}$ and $\boldsymbol{\beta}$ is the corresponding parameters to be estimated. $\varepsilon_{i}$ is the unobserved disturbance term. The traditional ordered logit model arises by assuming the disturbance term $\varepsilon_{i}$ to be identically and independently standard logistic distributed [34]. In the framework of traditional ordered logit model, the observed driver injury severity $Y_{i}$ can be mapped from $S_{i}$ through the threshold $\mu_{j}$,

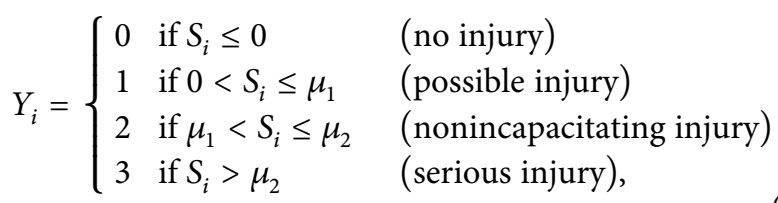

where, $\mu_{j}$ is the threshold for splitting the observed injury severity to be estimated and $\mu_{0}$ is normalized to 0 . The probability of observed injury severity outcomes for driver $i$ are described as,

$$
\operatorname{Prob}\left(Y_{i}=j\right)= \begin{cases}F\left(-\boldsymbol{\beta} \mathbf{X}_{i}\right) & \text { if } j=0 \\ F\left(\mu_{1}-\boldsymbol{\beta} \mathbf{X}_{i}\right)-F\left(-\boldsymbol{\beta} \mathbf{X}_{i}\right) & \text { if } j=1 \\ F\left(\mu_{2}-\boldsymbol{\beta} \mathbf{X}_{i}\right)-F\left(\mu_{1}-\boldsymbol{\beta} \mathbf{X}\right) & \text { if } j=2 \\ 1-F\left(\mu_{2}-\boldsymbol{\beta} \mathbf{X}_{i}\right) & \text { if } j=3,\end{cases}
$$

where, $F(\cdot)$ is the cumulative distribution function for the $\varepsilon_{i}$. The traditional ordered logit model assumes that the parameters are restricted to be fixed across individual observations, thereby failing to account for the unobserved individual heterogeneity. Therefore, the random parameters ordered logit model is developed, relaxing parameters to be randomly distributed with the formula as,

$$
\beta_{k}=\mu_{k}+\sigma_{k} v_{i k}
$$

where $\beta_{k}$ is the parameter of $k$ th indicator in vector $\beta, \mu_{k}$ and $\sigma_{k}$ are the mean and standard deviation of $\beta_{k}$, respectively. $v_{i k}$ is the individual specific heterogeneity, with mean zero and standard deviation one. The normal distribution is the typical distribution for specifying $v_{i k}$ in previous studies [21, 22], thus the normal distribution is considered to select the appropriate density function in this study. 
TABLE 1: Descriptions and summary statistics of variables.

\begin{tabular}{|c|c|c|c|}
\hline Variables & Description & $\begin{array}{c}\text { Passenger car } \\
\text { Count(percent) }\end{array}$ & $\begin{array}{c}\text { SUV } \\
\text { Count(percent) }\end{array}$ \\
\hline \multicolumn{4}{|l|}{ Response variable } \\
\hline \multirow{4}{*}{ Injury severity } & No injury & $1107(46.13)$ & $1102(46.69)$ \\
\hline & Possible injury & $515(21.46)$ & $436(18.47)$ \\
\hline & Nonincapacitating injury & $555(23.13)$ & $584(24.75)$ \\
\hline & Serious injury & $223(9.29)$ & $238(10.08)$ \\
\hline \multicolumn{4}{|l|}{ Discrete variables } \\
\hline \multirow{2}{*}{ Safety belt use } & Yes & $2134(88.92)$ & $2120(89.83)$ \\
\hline & $\mathrm{No}^{*}$ & $266(11.08)$ & $240(10.17)$ \\
\hline \multirow{2}{*}{ Airbag deployment } & Yes & $936(39.00)$ & $627(26.57)$ \\
\hline & $\mathrm{No}^{*}$ & $1464(61.00)$ & $1733(73.43)$ \\
\hline \multirow{2}{*}{ Drug or alcohol use } & Yes & $139(5.79)$ & $128(5.42)$ \\
\hline & $\mathrm{No}^{*}$ & $2261(94.21)$ & $2232(94.58)$ \\
\hline \multirow{2}{*}{ Failure to drive in single lane } & Yes & $409(17.04)$ & $367(15.55)$ \\
\hline & $\mathrm{No}^{*}$ & 1991(82.96) & 1993(84.45) \\
\hline \multirow{2}{*}{ Fatigue } & Yes & $205(8.54)$ & $137(5.81)$ \\
\hline & $\mathrm{No}^{*}$ & 2195(91.46) & 2223(94.19) \\
\hline \multirow{2}{*}{ Improper evasive action } & Yes & $446(18.58)$ & $537(22.75)$ \\
\hline & $\mathrm{No}^{*}$ & $1954(81.42)$ & $1823(77.25)$ \\
\hline \multirow{2}{*}{ Failure to control speed } & Yes & $324(13.50)$ & 231(9.79) \\
\hline & $\mathrm{No}^{*}$ & $2076(86.50)$ & $2129(90.21)$ \\
\hline \multirow{2}{*}{ Driver gender } & Male & $1433(59.71)$ & $1362(57.71)$ \\
\hline & Female* & $967(40.29)$ & $998(42.29)$ \\
\hline \multirow{3}{*}{ Ejection } & Yes & $103(4.29)$ & $110(4.66)$ \\
\hline & $\mathrm{No}^{*}$ & $2297(95.71)$ & $2250(95.34)$ \\
\hline & Turning left & $35(1.46)$ & $40(1.69)$ \\
\hline \multirow[t]{2}{*}{ Vehicle action } & Turning right & $33(1.38)$ & $39(1.65)$ \\
\hline & Going straight ${ }^{*}$ & 2332(97.17) & $2281(96.65)$ \\
\hline \multirow{2}{*}{ Intersection } & Yes & $127(5.29)$ & $97(4.11)$ \\
\hline & $\mathrm{No}^{*}$ & $2273(94.71)$ & 2263(95.89) \\
\hline \multirow{5}{*}{ Road class } & Interstate & $424(17.67)$ & $437(18.52)$ \\
\hline & US \& State highway & $792(33.00)$ & $779(33.01)$ \\
\hline & Farm to market road & $495(20.63)$ & $551(23.35)$ \\
\hline & County road & $356(14.83)$ & $355(15.04)$ \\
\hline & Others (city street, etc.) & $333(13.88)$ & $238(10.08)$ \\
\hline \multirow{3}{*}{ Road alignment } & Curve & $746(31.08)$ & $751(31.82)$ \\
\hline & Straight ${ }^{*}$ & $1654(68.92)$ & $1609(68.18)$ \\
\hline & No control $^{*}$ & $592(24.67)$ & $515(21.82)$ \\
\hline \multirow{3}{*}{ Traffic control type } & Signal/stop/yield/warning sign & $105(4.38)$ & $93(3.94)$ \\
\hline & Marked lanes & $930(38.75)$ & $956(40.51)$ \\
\hline & Center stripe/divider & $531(22.13)$ & $539(22.84)$ \\
\hline \multirow{13}{*}{ Month } & Other sign (human control, etc.) & $242(10.07)$ & $257(10.89)$ \\
\hline & January & $188(7.83)$ & $174(7.37)$ \\
\hline & February & $202(8.42)$ & $204(8.64)$ \\
\hline & Marth & $199(8.29)$ & $210(8.90)$ \\
\hline & April & $181(7.54)$ & $190(8.05)$ \\
\hline & May & 191(7.96) & $209(8.86)$ \\
\hline & June & $185(7.71)$ & $185(7.84)$ \\
\hline & July & $185(7.71)$ & $199(8.43)$ \\
\hline & August & $192(8.00)$ & $196(8.31)$ \\
\hline & September & $178(7.42)$ & $159(6.74)$ \\
\hline & October & $235(9.79)$ & $194(8.22)$ \\
\hline & November & $197(8.21)$ & $206(8.73)$ \\
\hline & December & $267(11.13)$ & $234(9.92)$ \\
\hline
\end{tabular}


TABLE 1: Continued.

\begin{tabular}{|c|c|c|c|c|c|c|c|c|}
\hline Variables & \multicolumn{4}{|c|}{ Description } & \multicolumn{2}{|c|}{$\begin{array}{c}\text { Passenger car } \\
\text { Count(percent) }\end{array}$} & \multicolumn{2}{|c|}{$\begin{array}{c}\text { SUV } \\
\text { Count(percent) }\end{array}$} \\
\hline & \multicolumn{4}{|c|}{ Sunday* } & \multicolumn{2}{|c|}{$429(17.88)$} & \multicolumn{2}{|c|}{$400(16.95)$} \\
\hline & \multicolumn{4}{|c|}{ Monday } & \multicolumn{2}{|c|}{$323(13.46)$} & \multicolumn{2}{|c|}{$317(13.43)$} \\
\hline & \multicolumn{4}{|c|}{ Tuesday } & \multicolumn{2}{|c|}{$260(10.83)$} & \multicolumn{2}{|c|}{$289(12.25)$} \\
\hline Day of week & \multicolumn{4}{|c|}{ Wednesday } & \multicolumn{2}{|c|}{$252(10.50)$} & \multicolumn{2}{|c|}{$271(11.48)$} \\
\hline & \multicolumn{4}{|c|}{ Thursday } & \multicolumn{2}{|c|}{$280(11.67)$} & \multicolumn{2}{|c|}{$281(11.91)$} \\
\hline & \multicolumn{4}{|c|}{ Friday } & \multicolumn{2}{|c|}{$402(16.75)$} & \multicolumn{2}{|c|}{$353(14.96)$} \\
\hline & \multicolumn{4}{|c|}{ Saturday } & \multicolumn{2}{|c|}{$454(18.92)$} & \multicolumn{2}{|c|}{$449(19.03)$} \\
\hline \multirow{3}{*}{ Time } & \multicolumn{4}{|c|}{ Peak hour } & \multicolumn{2}{|c|}{$441(18.38)$} & \multicolumn{2}{|c|}{$487(20.64)$} \\
\hline & \multicolumn{4}{|c|}{ Off-peak hour* } & \multicolumn{2}{|c|}{$834(34.75)$} & \multicolumn{2}{|c|}{$976(41.36)$} \\
\hline & & & t time & & & & 897( & $.01)$ \\
\hline & & & Jes & & & & 1675 & $0.97)$ \\
\hline Rural area & & & $\mathrm{Jo}^{*}$ & & & & 685( & $.03)$ \\
\hline & & & Tes & & & & 392( & $5.61)$ \\
\hline Adverse weather & & & $\mathrm{Jo}^{*}$ & & & & 1968 & 3.39) \\
\hline & & & Vet & & & & 602( & $.51)$ \\
\hline Road surface conditions & & & ry $^{*}$ & & & & 1758 & 4.49) \\
\hline & & & light & & & & 1360 & 7.68) \\
\hline & & & h/dusk & & & & 54( & 29) \\
\hline Light conditions & & Nigl & vith ligh & & & & 249 & $.56)$ \\
\hline & & Night & thout lig & & & & 695( & $.47)$ \\
\hline & & & nger car & & & SUV & & \\
\hline Continuous variables & Min & $\operatorname{Max}$ & Mean & S.D. & Min & $\operatorname{Max}$ & Mean & S.D. \\
\hline Age (years) & 15 & 97 & 29.600 & 13.339 & 15 & 87 & 32.014 & 13.984 \\
\hline Model year (years) & 0 & 27 & 15.738 & 5.199 & 0 & 27 & 13.132 & 4.564 \\
\hline Speed limit (mph) & 5 & 85 & 56.905 & 15.029 & 10 & 85 & 58.074 & 14.748 \\
\hline
\end{tabular}

Note: *base category; the actual vehicle model year ranges from 1990 to 2017 and the 1990 year was set as the base year, thus, the model year in Table 1 is the actual vehicle model year minus the base year (1990).

Since it is difficult to compute the probabilities, hence the random parameters ordered logit model is usually estimated by simulated maximum likelihood approaches (such as Monte Carlo simulation method). There have been considerable studies on how best to draw values of $\boldsymbol{\beta}$ so that accurate approximations of the probabilities are obtained with few draws as possible. For instance, Bhat [35] verified that Halton draws approach developed by Halton [36] was significantly more efficient than purely random draws and Halton draws have been widely used in previous studies [21,37].

Additionally, we need to test goodness-of-fit after estimation, a common measurement is Akaike information criterion (AIC), The AIC statistic is

$$
A I C=-2 L L(\beta)+2 K,
$$

where $L L(\boldsymbol{\beta})$ is $\log$-likelihood function of the estimated model at convergence and $K$ is the number of parameters in the estimated model.

3.2. Model Specification Tests. In order to demonstrate the superiority of the random parameters ordered logit (RPOL) model to the traditional ordered logit (TOL) model, the likelihood-ratio tests were implemented [31],

$$
\chi^{2}=-2\left[L L\left(\boldsymbol{\beta}_{T O L}\right)-L L\left(\boldsymbol{\beta}_{R P O L}\right)\right]
$$

where $L L\left(\boldsymbol{\beta}_{T O L}\right)$ and $L L\left(\boldsymbol{\beta}_{R P O L}\right)$ are the log-likelihood function of the TOL and RPOL models at convergence, respectively. The degrees of freedom for $\chi^{2}$ are the differences between the number of parameters of TOL and RPOL models.

In this study, driver injury severity in passenger car and SUV rollover crashes were modeled separately. To demonstrate the necessity of developing passenger car and SUV models separately, likelihood-ratio tests were conducted,

$$
\chi^{2}=-2\left[L L\left(\boldsymbol{\beta}_{\text {Full }}\right)-L L\left(\boldsymbol{\beta}_{\text {Passenger car }}\right)-L L\left(\boldsymbol{\beta}_{\text {SUV }}\right)\right],
$$

where $L L\left(\boldsymbol{\beta}_{\text {Full }}\right), L L\left(\boldsymbol{\beta}_{\text {Passenger car }}\right), L L\left(\boldsymbol{\beta}_{S U V}\right)$ are the log-likelihood function of the RPOL models at convergence for full (aggregated passenger cars and SUVs), passenger car, and SUV rollover crash data, respectively. The degrees of freedom for $\chi^{2}$ are the sum of the number of parameters in passenger car and SUV models minus the number of parameters in full model.

3.3. Analysis of Marginal Effects. In the random parameters ordered logit approach, parameters can only reflect the trends of influence of risk factors on driver injury severity, but cannot analyze the quantitative effects of explanatory variables on the dependent variable. Therefore, to further describe the impacts of risk factors on driver injury severity, the marginal effects for variables are calculated in this paper. For continuous variables, the marginal effects can be calculated as, 


$$
M_{X_{i k}}^{\operatorname{Prob}\left(Y_{i}=j\right)}=\frac{\partial \operatorname{Prob}\left(Y_{i}=j\right)}{\partial X_{i k}}
$$

$M_{X_{i k}}^{\operatorname{Prob}\left(Y_{i}=j\right)}$ represents the marginal effects of $k t h$ variable for individual $i$. The formula (8) is not applicable to 0 -1 indicators (or discrete variables), following the previous work undertaken by Kim et al. [21] and Agbelie [22], the marginal effects for $0-1$ indicators can be computed as,

$M_{X_{i k}}^{\operatorname{Prob}\left(Y_{i}=j\right)}=\operatorname{Prob}\left(Y_{i}=j\right)\left[X_{i k}=1\right]-\operatorname{Prob}\left(Y_{i}=j\right)\left[X_{i k}=0\right]$.

A marginal effect corresponds to the change in the probability of an outcome associated with a one-unit change in a covariate. Note that each individual has different marginal effects at different severity levels, thus in this study, the average marginal effects across the crash data were computed.

\section{Results and Discussion}

As shown in Table 1, a lot of independent variables were considered as underlying risk factors that associated with driver injury severity. Thus, to avoid the multi-collinearity problem in estimating the model, a multi-collinearity diagnosis using the variance inflation factors (VIF) method was conducted before specifying the model. As show in Table 2, the US \& State highway has the maximum VIF value with 4.70 in SUV sub dataset, which was far less than the threshold value (10). Therefore, diagnosis results implied that there was no strong multi-collinearity among independent variables in all three datasets.

To demonstrate the validity of the random parameters ordered logit model, the traditional ordered logit model was also developed. Thus, four models with two for passenger cars and the other two for SUVs were estimated. In addition, to justify the necessity of developing single-passenger car and SUV rollover crashes separately, the random parameters ordered logit and traditional ordered logit models were also developed for conducting the likelihood test. As such, a total of six models were estimated eventually. In all these models, variables that were statistically significant at the confidence of 95\% were considered to be significant. Notably, explanatory variables with a $t$-Statistic less than 1.96 were removed from the final model.

For the random parameters ordered logit models, at the beginning, all parameters were assumed to be normally distributed. Both mean and standard deviation of a random parameter estimate were tested whether they were statistically different zero at a given significance level. If the standard deviation around the mean is statistically equal to 0 , the random parameter was reduced to be fixed across observations. In order to determine appropriate Halton draws, a series of Halton draws (50, 100, 200, 500, and 1000 draws) have been investigated to estimate the models. Model results show that the estimated parameters and log-likelihood are indistinguishable when Halton draws reached to 200, which indicates that the model estimation tends to be stable. As such, this paper used 200 Halton draws to estimate the random parameters ordered logit models eventually.
TABLE 2: Summary statistic for VIF value of independent variables varying datasets.

\begin{tabular}{lccccc}
\hline Dataset & \multicolumn{2}{c}{ Min } & \multicolumn{2}{c}{ Max } & Mean \\
\hline $\begin{array}{l}\text { Passenger } \\
\text { car sub } \\
\text { dataset }\end{array}$ & Age & 1.04 & $\begin{array}{c}\text { US \& State } \\
\text { highway }\end{array}$ & 3.80 & 1.81 \\
$\begin{array}{l}\text { SUV sub } \\
\text { dataset }\end{array}$ & Age & 1.04 & $\begin{array}{c}\text { US \& State } \\
\text { highway }\end{array}$ & 4.70 & 1.92 \\
Full dataset & Age & 1.03 & $\begin{array}{c}\text { US \& State } \\
\text { highway }\end{array}$ & 4.12 & 1.84 \\
\hline
\end{tabular}

4.1. Model Comparison. The summary statistics for six models were shown in Table 3. The random parameters ordered logit models are superior to traditional ordered logit models in terms of AIC statistics and likelihood-ratio tests. More specifically, the AIC statistics in random parameters ordered logit models for passenger cars and SUVs are 24 and 23.7 points lower than that in traditional ordered logit models, respectively. Meanwhile, the likelihood-ratio tests for passenger car and SUV models are both significant at $0.5 \%$ level. In addition, the likelihood-ratio test for the necessity of developing passenger car and SUV models separately is significant at $0.5 \%$ level, implying it is rational to model driver injury severity in single-passenger car and SUV rollover crashes separately. Therefore, the following section shows the estimated parameter results and the impacts of significant risk factors associated with driver injury severity in singlepassenger car and SUV rollover crashes are described and discussed in the following subsections.

4.2. Results Analysis. Table 4 presents the estimation results in random parameters ordered logit models for passenger car and SUV crashes, respectively. In order to obtain quantitative effects for the variables on driver severity levels, the marginal effects for the statistically significant variables in the model were calculated (as shown in Table 5). The results clearly indicated the similarities and differences between passenger car and SUV models in terms of significant variables. A total of 14 safety factors were found to be significant in both models, such as male drivers, safety belt use, airbag deployment, ejection or partial ejection, drug or alcohol use, failure to drive in single lane, model year, speed limit, adverse weather conditions, rural area. Some variables such as driver's age, improper evasive action, turning right, intersection, Friday, August, and night with light are found to be significant only in one model.

A comparison of differences in the influence of safety factors that were significant in both models on nonincapacitating and serious injury outcomes in single-vehicle passenger car and SUV rollover crashes were conducted. As shown in Table 6, the marginal effect for the same variable specified to nonincapacitating injury or serious injury has the same sign in passenger car and SUV models. In addition, most of variables (such as male drivers, safety belt use) have stronger effects on nonincapacitating injury and serious injury outcomes in SUV than in passenger car rollover crashes. 
TABLE 3: Summary statistics and the likelihood test results for six models varying datasets.

\begin{tabular}{|c|c|c|c|c|c|c|}
\hline \multirow{2}{*}{ Dataset } & \multicolumn{2}{|c|}{ Passenger car } & \multicolumn{2}{|c|}{ SUV } & \multicolumn{2}{|c|}{ Full dataset } \\
\hline & TOL & RPOL & TOL & RPOL & TOL & RPOL \\
\hline Number of observations, $N$ & 2400 & 2400 & 2360 & 2360 & 4760 & 4760 \\
\hline Number of parameters, $K$ & 17 & 22 & 16 & 24 & 17 & 22 \\
\hline Log likelihood at zero, $L L(\mathbf{0})$ & -2991.7 & -2991.7 & -2937.1 & -2937.1 & -6168.4 & -6168.4 \\
\hline Log likelihood at convergence, $L L(\boldsymbol{\beta})$ & -2747.4 & -2730.4 & -2701.7 & -2681.9 & -5669.0 & -5651.8 \\
\hline $\mathrm{AIC}$ & 5528.8 & 5504.8 & 5435.4 & 5411.7 & 11372.0 & 11347.6 \\
\hline
\end{tabular}

Likelihood-ratio tests for the superiority of the RPOL model

$\chi^{2}=-2\left[L L\left(\boldsymbol{\beta}_{T O L}\right)-L L\left(\boldsymbol{\beta}_{R P O L}\right)\right]$

34

5

$<0.005$
39.6

8

$<0.005$

Likelihood-ratio tests for the necessity of developing passenger car and SUV models separately

$\chi^{2}=-2\left[L L\left(\boldsymbol{\beta}_{\text {Full }}\right)-L L\left(\boldsymbol{\beta}_{\text {Passenger car }}\right)-L L\left(\boldsymbol{\beta}_{S U V}\right)\right]$
Degrees of freedom

439.8

16

$<0.005$
479

24

$<0.005$

Note: TOL represents traditional ordered logit model, while RPOL represents random parameters ordered logit model, all TOL models have a constant.

Model results also found that a host of risk factors have heterogeneous effects on driver injury severity in singlevehicle passenger car and SUV rollover crashes. More specifically, six parameters (male drivers, drivers' age, airbag deployment, failure to drive in single lane, speed limit, and rural area) were found to be normally distributed in passenger car model, while nine variables (male drivers, safety belt use, airbag deployment, drug or alcohol use, failure to drive in single lane, improper evasive action, vehicle model year, Fridays, and rural area) were found to have a normally distributed parameter in SUV model. A further discussion on the significant variables is shown below.

4.2.1. Driver Characteristics. Turning to driver characteristics, the model results indicate that drivers gender is significantly associated with driver injury severity in rollover crashes. Male drivers are less likely to sustain serious injury by 1.53 and $2.18 \%$ in single-passenger car and SUV rollover crashes, respectively. Furthermore, empirical results show that male drivers in passenger car are normally distributed with mean -0.807 and standard deviation 1.406 , showing that about $71.7 \%$ of the population is below zero, and $28.3 \%$ of the population is above zero. This indicates that $71.7 \%$ of the male drivers decreases the risk of severe injury, while $28.3 \%$ of such a group elevates the probability of severe injury in passenger rollovers. The SUV model also captures similar results that male drivers have a normally distributed parameter with mean -1.145 and standard deviation 1.638. Thus implying that approximately half the male drivers are more likely to be injured severely, while the other half of male drivers have a lower probability to get involved in severe injury in SUV rollover crashes. This gender difference can be caused by some unseen factors including drivers height, weight, and perceptions of risk that are not included in the model.

Driver's age has significant impacts on driver injury severity in passenger car rollover crashes. The results show that older drivers are more likely to result in severe injuries (oneyear increase in age result in a $0.04 \%$ increase in the probability of serious injury for drivers in passenger cars). This finding reveals changes in demographic characteristics that population is aging. This variable is also found to be derived from a normal distribution and have a mean of 0.023 and a standard deviation of 0.031 , implying $22.9 \%$ of the population is less than 0 and $77.1 \%$ is greater than 0 . This indicates that most of the older drivers $(77.1 \%)$ increase the likelihood of fatality, while the other $22.9 \%$ of the older drivers decline the risk of fatality in passenger car rollovers. It is acknowledged that drivers physical abilities, reaction time, and driving behaviors can be varied in same ages [28]. Age is just a proxy for these unobserved factors, which may lead to different impacts of age on rollover crash injury severities among drivers within the same age group.

Safety belt use results in a $3.01 \%$ and $5.28 \%$ reduction in serious injury for drivers in passenger car and SUV rollover crashes, respectively. As noted in previous research, safety belt can protect against serious injuries, head injury, and brain injury, hence considerable efforts have been taken to examine the protective effect of safety belt $[16,17]$. In addition, safety belt use produces a normally distributed parameter with a mean -1.592 and standard deviation 0.712 in SUV model, implying $98.7 \%$ of drivers fastened safety belt are less likely to suffer severe injury outcomes, while $1.3 \%$ of safety belt users are more likely to be involved in severe injuries. This heterogenous effects could be caused by some uncover factors such as the service life of safety belt, driver's physical conditions, and etc.

A notable finding is that airbag deployment increases driver injury severity outcomes in both passenger car and SUV rollover crashes, which contradicts the traditional belief that airbag deployment can reduce injury severity outcomes. For instance, Wallis and Greaves [38] reviewed a series of studies about the relationship between airbag deployment and injuries in accidents, showing that airbags provide excellent protection against serious injury but are more likely to lead to minor injury. This disturbing result in the current study reflects the fact that the airbag fitted in motor vehicle is designed for 
TABLE 4: Estimation results for mixed ordered logit models.

\begin{tabular}{|c|c|c|c|c|c|c|}
\hline \multirow{2}{*}{ Variables } & \multicolumn{3}{|c|}{ Passenger car } & \multicolumn{3}{|c|}{ SUV } \\
\hline & Coef. & Std. Err. & $Z$ & Coef. & Std. Err. & $Z$ \\
\hline Male & -0.807 & 0.089 & -9.06 & -1.145 & 0.096 & -11.90 \\
\hline s.d. Male & 1.406 & 0.070 & 20.14 & 1.638 & 0.077 & 21.15 \\
\hline Age & 0.023 & 0.003 & 7.34 & & & \\
\hline s.d. Age & 0.031 & 0.002 & 19.41 & & & \\
\hline Safety belt use & -1.113 & 0.142 & -7.84 & -1.592 & 0.166 & -9.58 \\
\hline s.d. Safety belt use & & & & 0.712 & 0.077 & 21.15 \\
\hline Airbag deployment & 1.061 & 0.097 & 10.92 & 0.398 & 0.111 & 3.59 \\
\hline s.d. Airbag deployment & 0.559 & 0.070 & 7.98 & 0.644 & 0.089 & 7.26 \\
\hline Ejection or partial ejection & 4.025 & 0.278 & 14.50 & 3.959 & 0.301 & 13.17 \\
\hline Drug or alcohol use & 0.957 & 0.199 & 4.82 & 0.596 & 0.211 & 2.83 \\
\hline s.d. Drug or alcohol use & & & & 1.880 & 0.226 & 8.30 \\
\hline Failure to drive in single lane & 0.394 & 0.120 & 3.29 & 0.682 & 0.125 & 5.45 \\
\hline s.d. Failure to drive in single lane & 1.158 & 0.115 & 10.07 & 0.751 & 0.116 & 6.49 \\
\hline Improper evasive action & & & & 0.339 & 0.109 & 3.12 \\
\hline s.d. Improper evasive action & & & & 0.802 & 0.097 & 8.28 \\
\hline Turning right & -1.837 & 0.462 & -3.98 & & & \\
\hline Model year & -0.017 & 0.008 & -2.13 & -0.052 & 0.011 & -4.92 \\
\hline s.d. Model year & & & & 0.030 & 0.003 & 8.82 \\
\hline Intersection & & & & 0.384 & 0.060 & 6.37 \\
\hline Speed limit & 0.020 & 0.003 & 7.45 & 0.028 & 0.003 & 8.35 \\
\hline s.d. Speed limit & 0.009 & 0.001 & 11.65 & & & \\
\hline Friday & & & & -0.657 & 0.136 & -4.85 \\
\hline s.d. Friday & & & & 1.279 & 0.133 & 9.60 \\
\hline August & 0.436 & 0.165 & 2.64 & & & \\
\hline Adverse weather conditions & -0.683 & 0.144 & -4.74 & -0.681 & 0.128 & -5.31 \\
\hline Night with light & -0.500 & 0.134 & -3.74 & & & \\
\hline Rural area & -0.491 & 0.107 & -4.60 & -0.411 & 0.106 & -4.92 \\
\hline s.d. Rural area & 1.396 & 0.066 & 21.20 & 1.680 & 0.070 & 23.84 \\
\hline Threshold 1 & 1.594 & 0.064 & 24.96 & 1.437 & 0.063 & 22.79 \\
\hline Threshold 2 & 4.380 & 0.129 & 33.82 & 4.359 & 0.129 & 33.70 \\
\hline
\end{tabular}

horizontal collision and it is unlikely to trigger its deployment in a rollover [15]. However, airbag deployment produces a normally distributed parameter with a mean of 1.061 (or $0.398)$ and a standard deviation of $0.559(0.644)$ in passenger car (or SUV) model. 2.9\% (26.8\%) of the distribution is less than zero in passenger car model, indicating that the minority of drivers with airbag deployed sustained a lower probability of severe injury outcomes in both passenger car and SUV models.

Ejection or partial ejection increases driver injury severity outcomes in both passenger car and SUV rollover crashes. For example, ejection or partial ejection aggravate the likelihood of nonincapacitating injury by $29.59 \%$, and of serious injury by $44.05 \%$, but result in a lower of no injury by $44.15 \%$, and of possible injury by $29.49 \%$, which is in line with previous studies $[8,15,39,40]$. As these studies explained, casualties ejected from vehicles are more likely to collide with fixed objects or moving vehicles, thereby increasing the crash injury severity.

As can be expected, drivers involved with drugs or alcohol increase risk of possible injury by $0.38 \%$ (or $1.34 \%$ ), of nonincapacitating injury by $17.55 \%$ (or $11.05 \%$ ), and of serious injury by $2.54 \%$ (or $1.30 \%$ ) in passenger car (or SUV) rollovers. It can be explained that alcohol or drug impairs drivers recognition and reaction ability, which always leads to improper driving behaviors [17]. Failure to drive in single lane results in a higher likelihood of severe injury severity levels in both passenger car and SUV rollover crashes. Failure to drive in a single lane is one of the types of lane departure, which is a major consequence of rollovers. Elzen et al. [41] reported that about $90 \%$ of all rollovers in Europe and more than $40 \%$ of all fatalities in U.S. are caused by lane departure. Vehicle drifts out of its lane is an extremely dangerous behavior which tends to result in lane departure accidents and potentially fatal medical problems. As expected, drivers taking improper evasive action to deal with emergencies result in a slightly $(0.62 \%)$ higher probability in serious injury in SUV rollover crashes.

4.2.2. Vehicle and Road Attributes. Towards the vehicle and road attributes, by comparing with other vehicle actions including going straight, turning left, turning right contributes to a lower risk of possible injury by $22.15 \%$, of nonincapacitating injury by $16.79 \%$, and of serious injury by 
TABLE 5: Estimated marginal effects of safety factors in mixed ordered logit models (\%).

\begin{tabular}{|c|c|c|c|c|c|c|c|c|}
\hline \multirow{2}{*}{ Variables } & \multicolumn{4}{|c|}{ Passenger car } & \multicolumn{4}{|c|}{ SUV } \\
\hline & $\mathrm{NI}$ & PI & NII & SI & NI & PI & NII & SI \\
\hline Male & 19.07 & -4.49 & -13.05 & -1.53 & 26.91 & -5.10 & -19.63 & -2.18 \\
\hline Age & -0.57 & 0.16 & 0.36 & 0.04 & & & & \\
\hline Safety belt use & 23.62 & -0.27 & -20.34 & -3.01 & 31.48 & 4.35 & -30.55 & -5.28 \\
\hline Airbag deployment & -24.66 & 5.17 & 17.36 & 2.12 & -9.54 & 1.89 & 6.92 & 0.73 \\
\hline Ejection or partial ejection & -44.15 & -29.49 & 29.59 & 44.05 & -45.43 & -26.84 & 30.73 & 41.54 \\
\hline Drug or alcohol use & -20.47 & 0.38 & 17.55 & 2.54 & -13.69 & 1.34 & 11.05 & 1.30 \\
\hline Failure to drive in single lane & -9.28 & 1.97 & 6.55 & 0.77 & -15.57 & 1.82 & 12.48 & 1.45 \\
\hline Improper evasive action & & & & & -8.14 & 1.62 & 5.90 & 0.62 \\
\hline Turning right & 40.44 & -22.15 & -16.79 & -1.50 & & & & \\
\hline Model year & 0.41 & -0.12 & -0.27 & -0.03 & 1.28 & -0.32 & -0.88 & -0.09 \\
\hline Intersection & & & & & -9.41 & 2.33 & 6.44 & 0.64 \\
\hline Speed limit & -0.50 & 0.14 & 0.32 & 0.04 & -0.67 & 0.17 & 0.46 & 0.05 \\
\hline Friday & & & & & 16.27 & -5.63 & -9.75 & -0.89 \\
\hline August & -10.13 & 1.82 & 7.41 & 0.90 & & & & \\
\hline Adverse weather & 16.89 & -6.70 & -9.26 & -0.93 & 16.85 & -5.80 & -10.12 & -0.93 \\
\hline Night with light & 12.34 & -4.54 & -7.08 & -0.73 & & & & \\
\hline Rural area & 11.65 & -2.74 & -7.99 & -0.92 & 9.87 & -1.99 & -7.13 & -0.75 \\
\hline
\end{tabular}

NI: No injury, PI: Possible injury, NII: Nonincapacitating injury, SI: Serious injury.

TABLE 6: Comparison of selected variable marginal effects (\%).

\begin{tabular}{|c|c|c|c|c|c|c|}
\hline \multirow{2}{*}{ Variables } & \multicolumn{2}{|c|}{ Passenger car } & \multicolumn{2}{|c|}{ SUV } & \multicolumn{2}{|c|}{ Difference sign } \\
\hline & NII & SI & NII & SI & NII & SI \\
\hline Male & -13.05 & -1.53 & -19.63 & -2.18 & $\uparrow$ & $\uparrow$ \\
\hline Safety belt use & -20.34 & -3.01 & -30.55 & -5.28 & $\uparrow$ & $\uparrow$ \\
\hline Airbag deployment & 17.36 & 2.12 & 6.92 & 0.73 & $\downarrow$ & $\downarrow$ \\
\hline Ejection or partial ejection & 29.59 & 44.05 & 30.73 & 41.54 & $\uparrow$ & $\downarrow$ \\
\hline Drug or alcohol use & 17.55 & 2.54 & 11.05 & 1.3 & $\downarrow$ & $\downarrow$ \\
\hline Failure to drive in single lane & 6.55 & 0.77 & 12.48 & 1.45 & $\uparrow$ & $\uparrow$ \\
\hline Model year & -0.27 & -0.03 & -0.88 & -0.09 & $\uparrow$ & $\uparrow$ \\
\hline Speed limit & 0.32 & 0.04 & 0.46 & 0.05 & $\uparrow$ & $\uparrow$ \\
\hline Adverse weather & -9.26 & -0.93 & -10.12 & -0.93 & $\uparrow$ & $\uparrow$ \\
\hline Rural area & -7.99 & -0.92 & -7.13 & -0.75 & $\downarrow$ & $\downarrow$ \\
\hline
\end{tabular}

The difference sign is " " if the absolute value of marginal effects in SUV model is greater than that in passenger car model and "l" otherwise. For instance, for male variable specified to SI, the absolute values of marginal effects in passenger car and SUV models are $1.53 \%$ and $2.18 \%$, where $2.18 \%$ is greater than $1.53 \%$. Thus, the different sign is " $\uparrow "$.

$1.50 \%$ in passenger car rollover crashes. Consequently, two reasons might be responsible for this finding: (a) lower speed when turning right, and (b) less conflicts from the opposite directions. Vehicle model year is associated with driver injury severity significantly in both models, but it has a bigger impact on driver injury severity in SUV than in passenger car rollover crashes. This reflects the fact that the newer vehicles have better safety performance with the progress of science and technology. The model year of SUVs also produces a normally distributed parameter with a mean of -0.052 and a standard deviation of 0.030 , implying $4.2 \%$ of the population greater than zero. This means that $4.2 \%$ of drivers in the newer SUVs are more likely to sustain severe injury outcomes, which can be attributed to the difference in drivers physical conditions, vehicle use frequencies, and vehicle mileages.
In terms of speed limit, crashes occurred on roadways with a higher speed limit which would result in a higher probability of severe injury severity outcomes in both passenger car and SUV rollover crashes. For example, an increase of $1 \mathrm{mph}$ in speed limit will increase the probability of serious injury by $0.04-0.05 \%$ in passenger car and SUV crashes, respectively. This finding has been widely discussed in previous studies $[17,20]$. Notably, speed limits are found to be normally distributed parameter with mean -0.67 and standard deviation 16.27 in SUV rollover crashes, implying about $50 \%$ of drivers in SUVs are less likely to be involved in severe injury severity outcomes on roadways with high speed limits. The possible reason for this finding is that the mismatch between actual vehicle running speed and roadway speed limits. 
4.2.3. Environment Factors. Day of week also influences driver injury severity in SUV rollovers as indicated by the computed marginal effects. Rollover crashes occurring on Friday have a $16.27 \%$ greater probability of being no injury. In addition, this variable also results in a random parameter following a normally distribution with a mean of -0.657 and a standard deviation of 1.279 , indicating almost $70 \%$ of the distribution is below 0 , while $30 \%$ of the distribution is bigger than 0 . This result illustrates the complex driving behaviors of drivers after a week's work. Drivers face a higher probability of severe injury outcomes in August in both passenger car and SUV crashes, which is in line with previous studies [42].

Adverse weather conditions lead to a reduction of the likelihood of nonincapacitating injury by $9.26 \%$, and of serious injury by $0.93 \%$ in passenger car rollover crashes. Similar effects are also found in terms of SUV crashes that adverse weather conditions suppresses the risk of nonincapacitating injury (10.12\%) and incapacitating injury (0.93\%). This may be attributed to the fact that drivers are more cautious when driving in adverse weather conditions. Compared with other light conditions, drivers involved in passenger car crashes are $7.08 \%$ and $0.73 \%$ less likely to sustain nonincapacitating injury and serious injury in the night with light. The result can be ascribed to the possibility that drivers have better vision under light conditions [25].

Passenger car (or SUV) rollovers occurred in rural area has a $0.92 \%$ (or $0.75 \%$ ) lower probability of serious injury than in urban area. Again, the parameter of rural area in passenger car model (or SUV model) obeys a normal distribution with mean -0.491 (or 0.411) and standard deviation 1.396 (or 1.680), implying $36.3 \%$ and $40.3 \%$ drivers are more likely to suffer severe injury outcomes in passenger car and SUV rollover crashes, respectively. As verified by many studies, rural area is likely to result in severe injury severities. For example, Chen et al. [17] pointed out that rural roadways always have higher speed limits, hence the probability of speeding on rural roads are increased. The inexplicable result in the current study could be caused by some unobserved factors that are not included in the model or may be a finding specific to this dataset.

\section{Safety Implications}

Considering the risk factors which significantly related to driver injury severity, several potential safety countermeasures can be developed to mitigate injuries sustained by drivers in rollover crashes. The empirical research shows that failure to drive in single lane has a $18.16 \%$ higher likelihood of fatality, which implies that it is urgent to investigate a driving safety assistance system which can remind a driver to avoid leaving the lane. Previous studies strongly supported that the lane departure warming system is a safe and effective solution to prevent lane departure incident [43]. Thereby assembling a lane departure warming system for each vehicle before leaving the factory is an effective countermeasure to avoid lane departure accident. The research results also showed that drivers in the newer SUVs, on average, are less likely to sustain severe injury outcomes. The traffic management department should carry out strict annual inspection for the old SUVs on a regular basis.
Moreover, previous studies have strongly proven that wearing a safety belt effectively mitigate the prevalence of serious injuries or fatalities resulted in traffic accidents. The present study also shows that safety belt can reduce the likelihood of fatality in rollover crashes. However, Lorini et al. [44] reported that the safety belt wearing rates in low and middle-income countries ( $6 \%$ and $43 \%$, respectively) is lower relative to high-income countries (80\%). To achieve a high safety belt wearing rate, in the case of United States, three levels of effort can be conducted. First, the government should enact primary enforcement laws instead of secondary enforcement laws or no laws of safety belt. García-España et al. [45] addressed that primary enforcement laws are related to higher safety belt wearing rates, but merely half the states in United States have primary enforcement laws indeed. Second, the legal authorities need to strengthen the law enforcement and improve the penalties for driving without wearing safety belt. Third, public education programs targeted for all drivers and occupants are extensively needed to raise public awareness of using safety belt. With the popularity of smart phones, people can get any information they want from smart phones. Through integrating multiple safety educational resources from Internet and implanting it into mobile APP, people can learn traffic safety knowledge whenever and wherever possible from the online platform.

Furthermore, the analysis shows that the occurrence of rollovers in rural area has a $18.27 \%$ higher likelihood of incapacitating injury than in urban area. Reducing the speed limit might be a cost-effective countermeasure to mitigate crash injury severity in rural area. Meanwhile, fully popularizing the vehicle active safety technology such as intelligent speed assistance and independent emergency development to avoid accidents through active speed limit. Extra efforts such as adding more roadside warning signs, upgrading the road system and setting more reflective pavement markings are also needed to prevent injuries in rural area. Finally, some other efficiently proven programs, including license suspension laws, lower blood alcohol content (BAC) laws, enforcement of speed violation and roadway lighting, can also be undertaken to mitigate crash injuries [46].

\section{Conclusion and Future Research Directions}

The present study examined the contributing factors as well as their heterogeneous impacts on driver injury severity in single-vehicle passenger car and SUV rollover crashes separately. Using detailed policed data from Texas Department of Transportation in 2016, the random parameters ordered logit models were developed for analyzing the driver injury severity conditional on rollover crashes occurrence. The models were estimated by simulated maximum likelihood approaches with 200 Halton draws. The likelihood-ratio tests results showed that the random parameters ordered logit models are superior to traditional ordered logit models and it is necessary to model driver injury severity in passenger car and SUV rollover crashes separately. The model estimation results clearly indicated the similarities and differences between passenger car and SUV models in terms of safety factors associated with 
driver injury severity. A total of 14 safety factors were found to be significant in both models, such as male drivers, safety belt use, airbag deployment, ejection or partial ejection, drug or alcohol use, failure to drive in a single lane, model year, speed limit, adverse weather conditions, rural area. Some risk factors such as driver's age, improper evasive action, turning right, intersection, Friday, August, and night with light are found to be significant only in one model.

The results also showed that several risk factors lead to unobserved heterogeneity, with normally distributed parameters being affected by such heterogeneous effects. More specifically, six variables (male drivers, drivers' age, airbag deployment, failure to drive in single lane, speed limit, and rural area) were found to produce random parameters in passenger car model, while nine parameters (male drivers, safety belt use, airbag deployment, drug or alcohol use, failure to drive in single lane, improper evasive action, vehicle model year, Friday, and rural area) in SUV model were found to be random. In order to address unobserved heterogeneity issue due to these variables with random parameters, new data resources need to be used to supplement single crash data source, thereby adding more variables into the model in the future research. For example, more detailed information of drivers such as height, weight, physical abilities, etc. can be obtained from medical system or motor vehicle management system and can be integrated into crash data for safety analysis.

Another significant finding is that airbag deployment is associated with increased driver injury severity outcomes in both passenger car and SUV rollover crashes. A probable explanation is that airbag is not designed for rollovers but for horizontal collisions. Thus, twofold research efforts can be undertaken in the future: (a) conducting automobile flipping experiment to test whether the airbag can decrease injury severity of occupants in a rollover; (b) assessing the effects of airbag deployment on injury severity of turnover crashes and horizontal collisions separately to confirm whether the airbag's protective effects only valid for horizontal collisions.

In this study, development of the random parameters ordered logit model for analyzing driver injury severity in single-vehicle passenger car and SUV rollover crashes in Texas has led to a deep insight into causality nature and factor involved of driver injury severity. These findings are helpful for transport agencies determine effective countermeasures aimed at mitigating injuries sustained by occupants in single-vehicle rollover accidents. However, this paper also has some limitations. Firstly, the current research used the oneyears' worth crash dataset in one-state because it can control temporal and spatial heterogeneity to a certain degree. However, future research based on cross-temporal and cross-regional crash data is strongly recommended to provide more robust estimations. Secondly, the random parameters ordered logit model in this study characterized the mean and variance of random parameters by giving a distribution and captures heterogeneity only in the mean, which may not fully track the unobserved heterogeneity. Some of recent studies on the application of the random parameters logit model in analysis of crash injury severities provided much more flexibility in capturing the unobserved heterogeneity by allowing for the heterogeneity in means and variances of parameters $[47,48]$. Therefore, future research by extending simple random parameters to include heterogeneity in the means and variances would better account for unobserved heterogeneity in modeling driver injury severity in SUV and passenger car rollover crashes.

\section{Data Availability}

The crash data used to support the findings of this study were supplied by Texas Department of Transportation (TXDOT) under license and so cannot be made freely available. Requests for access to these data should be made to [https://cris.txdot.gov].

\section{Conflicts of Interest}

The authors declare no conflict of interest.

\section{Authors' Contributions}

Huiying Wen put forwarded the idea of the paper; Zuogan Tang estimated the model and wrote the paper; Yuchen Zeng collected and analyzed the data; Kexiong Zhang made editing corrections.

\section{Funding}

This research was funded by National Natural Science Foundation of China, grant numbers 51378222 and 51578247.

\section{Acknowledgments}

The authors would like to thank the Texas Department of Transportation for providing the crash data used in the current research.

\section{References}

[1] WHO, World Health Statistics 2017: Monitoring Health for the SDGs, Sustainable Development Goals, World Health Organization, Geneva, 2017.

[2] NHTSA, Traffic Safety Facts 2014: A Compilation of Motor Vehicle Crash Data from the Fatality Analysis Reporting System and the General Estimates System, National Highway Traffic Safety Administration, Washington, DC, USA, 2014.

[3] J. Pai, Trends and Rollover-Reduction Effectiveness of Static Stability Factor in Passenger Vehicles, National Highway Traffic Safety Administration, Washington, DC, USA, 2017.

[4] NBSC, China Road Traffic Accident Statistics 2016, National Bureau of Statistics of China, Beijing, China, 2016.

[5] TXDOT, Texas Motor Vehicle Traffic Crash Facts Calendar Year 2016, Texas Department of Transportation, Austin, TX, USA, 2016.

[6] C. M. Farmer and A. K. Lund, "Rollover risk of cars and light trucks after accounting for driver and environmental factors," Accident Analysis \& Prevention, vol. 34, no. 2, pp. 163-173, 2002. 
[7] A. Khattak, R. Schneider, and F. Targa, "Risk factors in large truck rollovers and injury severity: analysis of single-vehicle collisions," in Transportation Research Board 82nd Annual Meeting, Washington, DC, USA, 2002.

[8] A. Khattak and M. Rocha, "Are SUVs "supremely unsafe vehicles?": analysis of rollovers and injuries with sport utility vehicles," Journal of the Transportation Research Board, vol. 1840, no. 1, pp. 167-177, 2003.

[9] D. F. Huelke, T. E. Lawson, and J. C. Marsh, "Injuries, restraints and vehicle factors in rollover car crashes," Accident Analysis \& Prevention, vol. 9, no. 2, pp. 93-107, 1977.

[10] M. Keall and S. Newstead, "Induced exposure estimates of rollover risk for different types of passenger vehicles," Traffic Injury Prevention, vol. 10, no. 1, pp. 30-36, 2009.

[11] V. Rondelli, C. Casazza, and R. Martelli, "Tractor rollover fatalities, analyzing accident scenario," Journal of Safety Research, vol. 67, no. 12, pp. 99-106, 2018.

[12] S. M. Antunes, C. Cordeiro, and H. M. Teixeira, "Analysis of fatal accidents with tractors in the Centre of Portugal: ten years analysis," Forensic Science International, vol. 287, no. 6, pp. 74-80, 2018.

[13] M. Hosseinpour, A. S. Yahaya, A. F. Sadullah, N. Ismail, and S. M. R. Ghadiri, "Evaluating the effects of road geometry, environment, and traffic volume on rollover crashes," Transport, vol. 31, no. 2, pp. 221-232, 2016.

[14] M. C. Lo, R. P. Giffin, K. A. Pakulski, W. S. Davis, S. A. Bernstein, and D. V. Wise, "High-mobility multipurpose wheeled vehicle rollover accidents and injuries to U.S. Army soldiers by reported occupant restraint use, 1992-2013," Military Medicine, vol. 182, no. 5, pp. e1782-e1791, 2017.

[15] C. Conroy, D. B. Hoyt, A. B. Eastman et al., "Rollover crashes: predicting serious injury based on occupant, vehicle, and crash characteristics," Accident Analysis \& Prevention, vol. 38, no. 5, pp. 835-42, 2006.

[16] W. Hu and E. T. Donnell, "Severity models of cross-median and rollover crashes on rural divided highways in Pennsylvania," Journal of Safety Research, vol. 42, no. 5, pp. 375-382, 2011.

[17] C. Chen, G. Zhang, Z. Qian, R. A. Tarefder, and Z. Tian, "Investigating driver injury severity patterns in rollover crashes using support vector machine models," Accident Analysis \& Prevention, vol. 90, pp. 128-139, 2016.

[18] Q. Wu, G. Zhang, C. Chen, R. Tarefder, H. Wang, and H. Wei, "Heterogeneous impacts of gender-interpreted contributing factors on driver injury severities in single-vehicle rollover crashes," Accident Analysis \& Prevention, vol. 94, pp. 28-34, 2016.

[19] S. Islam, A. B. Hossain, and T. E. Barnett, "Comprehensive injury severity analysis of suv and pickup truck rollover crashes: Alabama case study," Journal of the Transportation Research Board, vol. 2601, pp. 1-9, 2016.

[20] A. J. Anarkooli, M. Hosseinpour, and A. Kardar, "Investigation of factors affecting the injury severity of single-vehicle rollover crashes: a random-effects generalized ordered probit model," Accident Analysis \& Prevention, vol. 106, pp. 399-410, 2017.

[21] J. K. Kim, G. F. Ulfarsson, S. Kim, and V. N. Shankar, "Driverinjury severity in single-vehicle crashes in California: a mixed logit analysis of heterogeneity due to age and gender," Accident Analysis \& Prevention, vol. 50, pp. 1073-1081, 2013.

[22] B. R. D. K. Agbelie, "The effect of gender on two-passenger vehicle highway crash-injury severity: a mixed logit empirical analysis," Journal of Transportation Safety and Security, vol. 8, no. 3, pp. 280-291, 2016.

[23] Q. Zeng, H. Wen, H. Huang, X. Pei, and S. C. Wong, "Incorporating temporal correlation into a multivariate random parameters tobit model for modeling crash rate by injury severity," Transportmetrica A: Transport Science, vol. 14, no. 3, pp. 177-191, 2018.

[24] Y. Xie, K. Zhao, and N. Huynh, "Analysis of driver injury severity in rural single-vehicle crashes," Accident Analysis \& Prevention, vol. 47, pp. 36-44, 2012.

[25] M. S. Shaheed and K. Gkritza, "A latent class analysis of singlevehicle motorcycle crash severity outcomes," Analytic Methods in Accident Research, vol. 2, pp. 30-38, 2014.

[26] Y. Xiong, J. L. Tobias, and F. L. Mannering, "The analysis of vehicle crash injury-severity data: a markov switching approach with road-segment heterogeneity," Transportation Research Part B: Methodological, vol. 67, pp. 109-128, 2014.

[27] N. V. Malyshkina and F. L. Mannering, "Markov switching multinomial logit model: an application to accident-injury severities," Accident Analysis \& Prevention, vol. 41, no. 4, pp. 829-838, 2009.

[28] F. L. Mannering, V. Shankar, and C. R. Bhat, "Unobserved heterogeneity and the statistical analysis of highway accident data," Analytic Methods in Accident Research, vol. 11, pp. 1-16, 2016.

[29] N. Venkataraman, V. Shankar, G. F. Ulfarsson, and D. Deptuch, "A heterogeneity-in-means count model for evaluating the effects of interchange type on heterogeneous influences of interstate geometrics on crash frequencies," Analytic Methods in Accident Research, vol. 2, pp. 12-20, 2014.

[30] TXDOT, Annual Motor Vehicle Crash Data Report Definitions, Texas Department of Transportation, Austin, TX, USA, 2016.

[31] F. Chang, M. Li, P. Xu, H. Zhou, M. Haque, and H. Huang, "Injury severity of motorcycle riders involved in traffic crashes in Hunan, China: a mixed ordered logit approach," International Journal of Environmental Research and Public Health, vol. 13, no. 7, p. 714, 2016.

[32] Q. Zeng, W. Gu, X. Zhang, H. Wen, J. Lee, and W. Hao, "Analyzing freeway crash severity using Bayesian spatial generalized ordered logit model with conditional autoregressive priors," Accident Analysis \& Prevention, vol. 127, pp. 87-95, 2019.

[33] F. Chang, P. Xu, H. Zhou, A. H. S. Chan, and H. Huang, "Investigating injury severities of motorcycle riders: a two-step method integrating latent class cluster analysis and random parameters logit model," Accident Analysis \& Prevention, vol. 131, no. 10, pp. 316-326, 2019.

[34] H. Naji, Q. Xue, N. Lyu, C. Wu, and K. Zheng, "Evaluating the driving risk of near-crash events using a mixed-ordered logit model," Sustainability, vol. 10, no. 8, pp. 1-20, 2018.

[35] C. R. Bhat, "Simulation estimation of mixed discrete choice models using randomized and scrambled Halton sequences," Transportation Research Part B: Methodological, vol. 37, no. 9, pp. 837-855, 2003.

[36] J. H. Halton, "On the efficiency of certain quasi-random sequences of points in evaluating multi-dimensional integrals," Numerische Mathematik, vol. 2, no. 1, pp. 84-90, 1960.

[37] Q. Wu, G. Zhang, X. Zhu, X. C. Liu, and R. Tarefder, "Analysis of driver injury severity in single-vehicle crashes on rural and urban roadways," Accident Analysis \& Prevention, vol. 94, pp. 35-45, 2016. 
[38] L. A. Wallis and I. Greaves, "Injuries associated with airbag deployment," Emergency Medicine Journal, vol. 19, no. 6, pp. 490-493, 2002.

[39] A. Donelson, K. Ramachandran, K. Zhao, and A. Kalinowski, "Rates of occupant deaths in vehicle rollover: importance of fatality-risk factors," Journal of Transportation Research Record, vol. 1665, no. 1, pp. 109-117, 1999.

[40] P. Albertsson, T. Falkmer, A. Kirk, E. Mayrhofer, and U. Björnstig, "Case study: 128 injured in rollover coach crashes in Sweden-Injury outcome, mechanisms and possible effects of seat belts," Safety Science, vol. 44, no. 2, pp. 87-109, 2006.

[41] C. V. D. Elzen and I. E. Fernandes Valeo, Embedded CameraBased System Counters Lane Departures, Datasheets Com, San Francisco, CA, USA, 2005.

[42] M. S. Shaheed and K. Gkritza, "A latent class analysis of singlevehicle motorcycle crash severity outcomes," Analytic Methods in Accident Research, vol. 2, pp. 30-38, 2014.

[43] B. Yu, W. Zhang, and Y. Cai, "A lane departure warning system based on machine vision," in IEEE Pacific-Asia Workshop on Computational Intelligence and Industrial Application, pp. 197-201, IEEE, Wuhan, China, 2008.

[44] C. Lorini, F. Pieralli, A. Mersi et al., "Comparison of selfreported and observed prevalence of safety belt and helmet use in Florence," Annali di Igiene: Medicina Preventiva E di Comunita, vol. 26, no. 6, pp. 499-506, 2014.

[45] J. F. García-España, F. K. Winston, and D. R. Durbin, "Safety belt laws and disparities in safety belt use among US high-school drivers," American Journal of Public Health, vol. 102, no. 6, pp. 1128-1134, 2012.

[46] S. N. Forjuoh, "Traffic-related injury prevention interventions for low-income countries," International Journal for Consumer \& Product Safety, vol. 10, no. 1-2, pp. 109-118, 2003.

[47] A. Behnood and F. Mannering, "Determinants of bicyclist injury severities in bicycle-vehicle crashes: a random parameters approach with heterogeneity in means and variances," Analytic Methods in Accident Research, vol. 16, pp. 35-47, 2017.

[48] P. Seraneeprakarn, S. Huang, V. Shankar, F. Mannering, N. Venkataraman, and J. Milton, "Occupant injury severities in hybrid-vehicle involved crashes: a random parameters approach with heterogeneity in means and variances," Analytic Methods in Accident Research, vol. 15, pp. 41-55, 2017. 


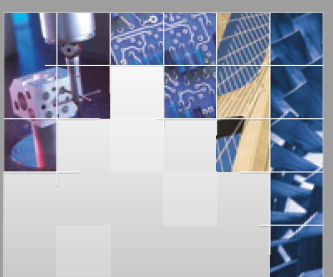

\section{Enfincering}
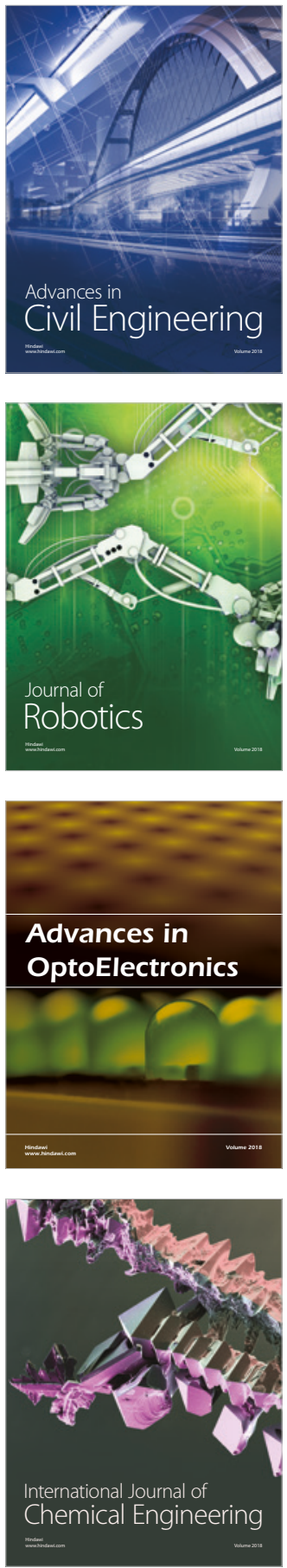

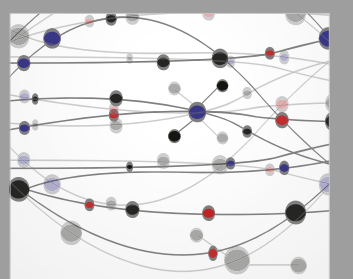

\section{Rotating \\ Machinery}

The Scientific World Journal

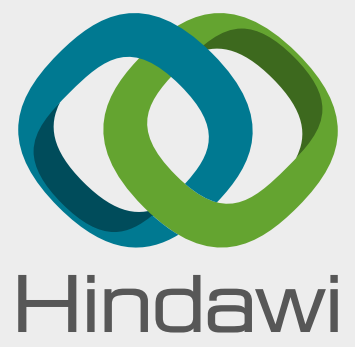

Submit your manuscripts at

www.hindawi.com
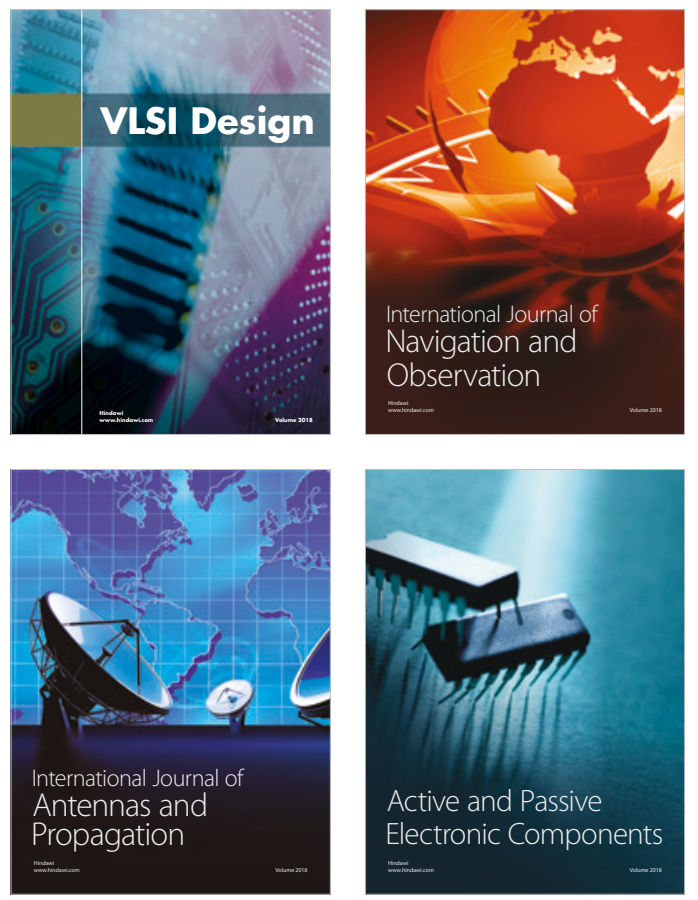
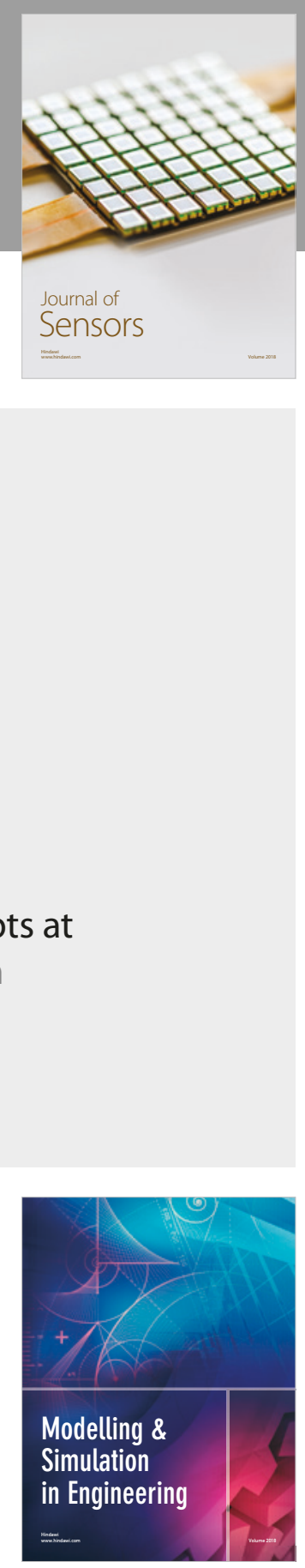

\section{Advances \\ Multimedia}
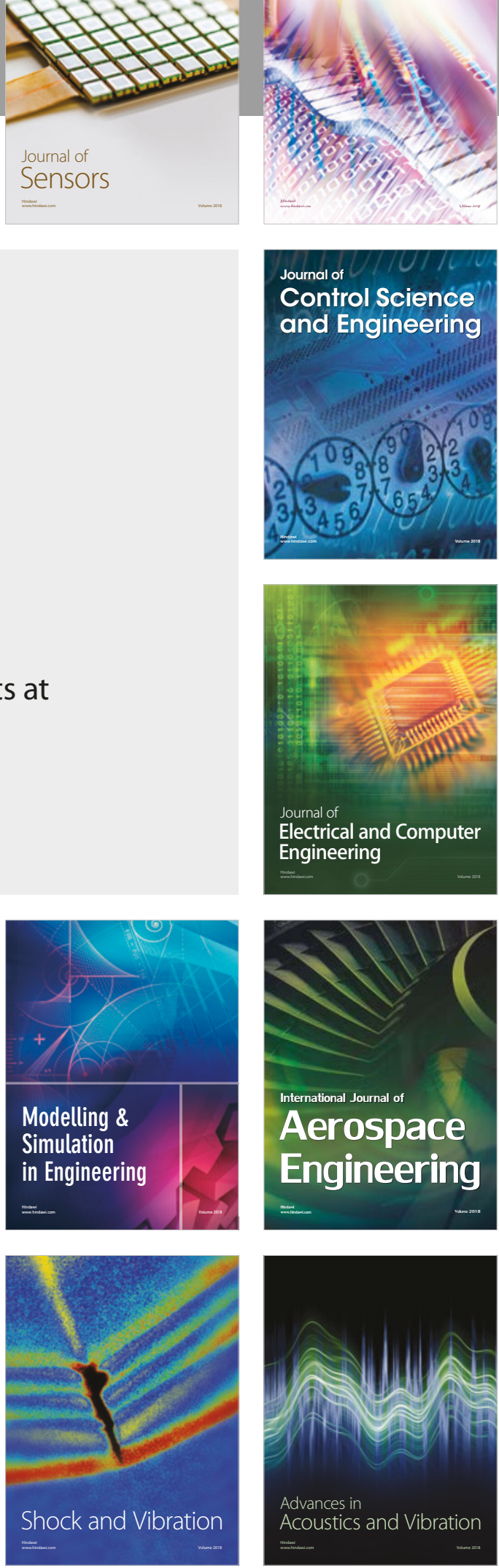